\title{
Proteinases in cardiovascular aneurysms and rupture: targets for therapy?
}

\author{
Peter Carmeliet \\ The Center for Transgene Technology and Gene Therapy, Flanders Interuniversity Institute for Biotechnology, \\ KU Leuven, Leuven, Belgium \\ Address correspondence to: P. Carmeliet, Center for Transgene Technology and Gene Therapy, \\ Campus Gasthuisberg, Herestraat 49, University of Leuven, B-3000 Leuven, Belgium. \\ Phone: 32-16-34-57-72; Fax: 32-16-34-59-90; E-mail: peter.carmeliet@med.kuleuven.ac.be.
}

The extracellular matrix plays an essential role in the integrity of the cardiovascular system. Under normal conditions, elastin and collagen fibers resist spontaneous breakdown and can only be degraded by matrix metalloproteinases (MMPs) or, indirectly, by plasminogen activators (PAs). Because these proteinases are potent, their activity in vivo requires tight control. Hence, they are secreted as inactive zymogens that can be activated by other proteinases, such as plasmin. In addition, their activity is neutralized by proteinase inhibitors endogenous to the various tissues. Activated inflammatory cells, which are rich sources of collagenolytic and elastolytic proteinases, are absent in the noninflamed vessel and heart. Under resting conditions, therefore, proteinase inhibitors predominate over activators in the cardiovascular system (Figure 1a). During inflammation, the proteolytic balance tilts and the resultant net proteolysis causes erosion, fragmentation, and dissolution of the elastic laminae and collagen bundles (Figure 1a). This pattern of events may occur acutely or sub-acutely after organ transplantation $(1,2)$ or myocardial infarction (3) or more chronically during atherosclerosis (4).

Rupture of an abdominal aortic aneurysm accounts for $2 \%$ of all deaths in men over 60 years of age (5), while cardiac rupture accounts for 5 to $31 \%$ of in-hospital mortality after acute myocardial infarction in middle-aged patients (6). Both disorders are unpredictable, are usually fatal, and have few available treatments $(5,6)$. Remarkably, genetic predisposition factors or criteria for identifying patients at risk for aortic or cardiac rupture remain largely undetermined. A better understanding of the mechanisms might allow these catastrophic events to be prevented, but the absence of reliable animal models has blocked progress in this field (7).
Indirect evidence suggests that the plasminogen system, including the activators urokinase-type PA (u-PA) and tissue-type PA (t-PA) and the plasminogen activator inhibitor PAI-1 as well as the MMPs, contributes to the formation of aortic, cerebral, and cardiac aneurysms (8-11). Based on in vitro activities and in vivo expression data, MMP-9 (gelatinase B) and MMP-12 (metalloelastase) in particular have been presumed to play a role. In this issue of the JCI, Pyo et al. (12) provide direct genetic evidence that MMP-9 plays an essential role in a mouse model formation. In their system, perfusion of pancreatic elastase into the aorta did not cause sufficient degradation of elastin fibers for aneurysmal dilation. Rather, it generated chemotactic matrix degradation products that induced a strong of nonatherosclerotic aortic aneurysm

inflammatory infiltration, leading to aneurysm formation. In contrast to wildtype mice, aortas in MMP-9-deficient mice did not undergo aneurysmal dilation, despite the fact that they developed a comparable inflammatory infiltration. Surprisingly, MMP-12-deficient mice were not protected. However, MMP-12 appeared to cooperate with MMP-9 in vessel wall destruction, as mice lacking both proteinases enjoyed greater protection from aneurysmal pathogenesis.

This study contributes in several respects to our understanding of cardiovascular proteinases. First, it provides direct genetic evidence for an involvement of MMPs - in particular, for the predominant role of MMP-9 in aneurysm formation, albeit in a nonatherosclerotic aneurysm model. Second, it highlights tissue-specific
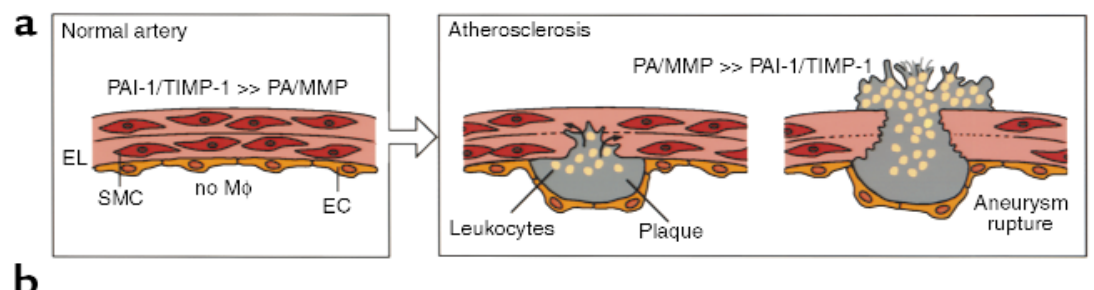

b

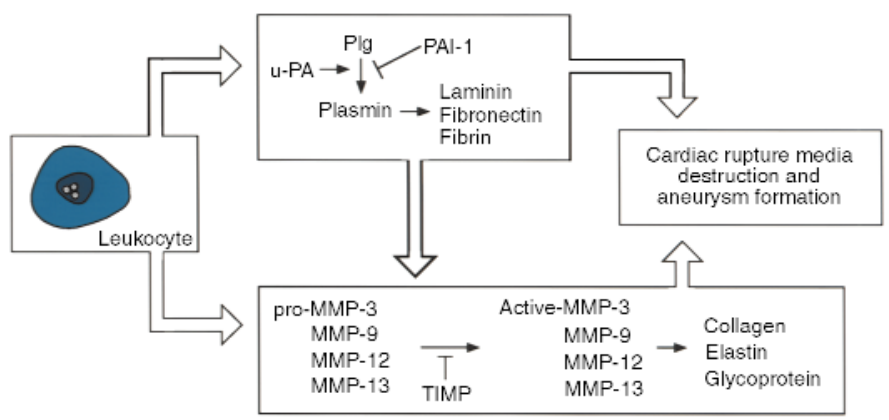

Figure 1

(a) Proteinase inhibitors predominate in the quiescent vessel, while net proteolysis degrades the media and causes aneurysmal rupture during atherosclerosis. EC, endothelial cell; SMC, smooth muscle cell; M $\phi$, macrophage; EL, elastic lamina. (b) Inflammatory cells produce plasminogen (Plg) activators that activate plasmin, which degrades fibrin, laminin, and fibronectin. By activating zymogen pro-MMPs, plasmin orchestrates degradation of collagen and elastin, leading to complete destruction of all vessel wall matrix components. 
roles for the different MMPs. Thus, it appears that MMP-12 is more important in pulmonary emphysema than in aneurysm formation. So, targeting MMP-12 activity might prove therapeutic for pulmonary disorders without causing significant adverse effects on the cardiovascular system. Third, it provides proof of principle that inhibitors of MMPs, especially MMP-9, could be used to prevent aortic aneurysms and cardiac rupture. Thus, it extends previous reports of a protective effect of the inhibition of MMPs or the overexpression of either PAI-1 or tissue inhibitor of metalloproteinases-1 (3, 13-15). Finally, it provides insight into the molecular basis of aneurysm formation. This study should provide the impetus for conducting genetic screens to identify defects in MMP9 or other proteinase genes that might predispose to destructive cardiovascular disorders.

A number of questions remain. First, is MMP-9 the therapeutic target of choice? MMP-9-deficient mice are protected against aneurysmal dilation, as seen in this study (12), and also from cardiac rupture (3). However, MMP-9 can degrade collagen only after initial cleavage by interstitial collagenases. Therefore, some degree of collagenolysis and tissue weakening probably occurs even in MMP-9-deficient mice, although the residual cleavage is apparently insufficient to cause aneurysmal dilatation. uPA-deficient mice are completely protected from rupture of atherosclerotic aortic aneurysms and ischemic myocardium and show reduced activation of MMP-9 (3, 4). Since u-PA and plasmin also lead to activation of MMPs $-3,-12$, and -13 , u-PA orchestrates the activation of an entire cascade of downstream MMP effectors (Figure 1b). Hence, u-PA and interstitial collagenases might present alternative, perhaps preferable, targets. Second, dilation of the aorta requires elastolysis, whereas rupture of the vessel wall depends on collagenolysis. In the present experimental system, rupture does not occur, so it remains uncertain whether MMP-9 contributes to this life-threatening event. Third, would therapeutic use of proteinase inhibitors cause harmful side effects? Proteinases participate in reproduction, wound healing, and angiogenesis, among many other essential processes. In addition, prolonged administration of proteinase inhibitors can promote cardiac failure by impairing healing and therapeutic angiogenesis after infarction (3). Despite this concern, the compelling evidence from this study that proteinases act in aneurysmal pathogenesis may suggest clinical applications for proteinase inhibitors in blocking progression of these fatal disorders.

1. Moons, L., et al. 1998. Reduced transplant arteriosclerosis in plasminogen-deficient mice. J. Clin. Invest. 102:1788-1797.

2. Allaire, E., et al. 1996. Cell and extracellular matrix rejection in arterial concordant and discordant xenografts in the rat. Transplantation. 62:794-803.

3. Heymans, S., et al. 1999. Inhibition of plasmino- gen activators or matrix metalloproteinases prevents cardiac rupture but impairs therapeutic angiogenesis and causes cardiac failure. Nat. Med. 5:1135-1142.

4. Carmeliet, P., et al. 1997. Urokinase-generated plasmin activates matrix metalloproteinases during aneurysm formation. Nat. Genet. 17:439-444.

5. Thompson, R.W. 1996. Basic science of abdominal aortic aneurysms: emerging therapeutic strategies for an unresolved clinical problem. Curr. Opin. Cardiol. 11:504-518.

6. Dalrymple-Hay, M.J., Monro, J.L., Livesey, S.A., and Lamb, R.K. 1998. Postinfarction ventricular septal rupture: the Wessex experience. Semin. Thorac. Cardiovasc. Surg. 10:111-116.

7. Carrell, T.W., Smith, A., and Burnand, K.G. 1999. Experimental techniques and models in the study of the development and treatment of abdominal aortic aneurysm. Br.J. Surg. 86:305-312.

8. Lupu, F., et al. 1995. Plasminogen activator expression in human atherosclerotic lesions. Arterioscler. Thromb. Vasc. Biol. 15:1444-1455.

9. Irizarry, E., et al. 1993. Demonstration of interstitial collagenase in abdominal aortic aneurysm disease. J. Surg. Res. 54:571-574.

10. Curci, J.A., Liao, S., Huffman, M.D., Shapiro, S.D and Thompson, R.W. 1998. Expression and localization of macrophage elastase in abdominal aortic aneurysms. J. Clin. Invest. 102:1900-1910.

11. Rohde, L.E., et al. 1999. Matrix metalloproteinase inhibition attenuates early left ventricular enlargement after experimental myocardia infarction in mice. Circulation. 99:3063-3070.

12. Pyo, R., et al. 2000. Targeted gene disruption of matrix metalloproteinase-9 (gelatinase B) suppresses development of experimental abdominal aortic aneurysms. J. Clin. Invest. 105:1641-1649.

13. Allaire, E., Forough, R., Clowes, M., Starcher, B. and Clowes, A.W. 1998. Local overexpression of TIMP-1 prevents aortic aneurysm degeneration and rupture in a rat model. J. Clin. Invest. 102:1413-1420

14. Allaire, E., et al. 1998. Prevention of aneurysm development and rupture by local overexpression of plasminogen activator inhibitor-1. Circulation. 98:249-255.

15. Thompson, R.W., and Baxter, B.T. 1999. MMP inhibition in abdominal aortic aneurysms. Rationale for a prospective randomized clinical trial. Ann. NY Acad. Sci. 878:159-178. 